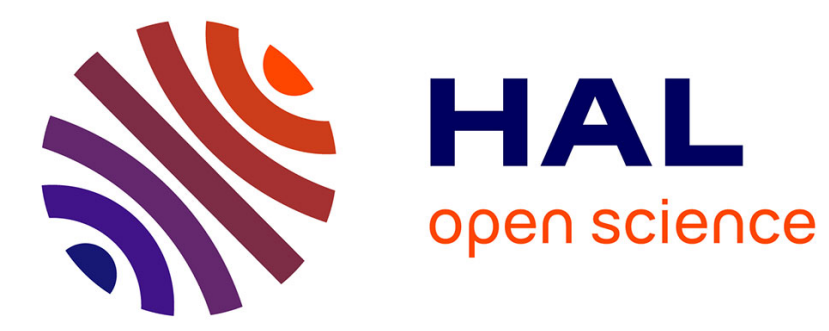

\title{
Unidirectional Thermal Effects in Current-Induced Domain Wall Motion
}

J Torrejon, Grégory Malinowski, M Pelloux, R Weil, A Thiaville, J Curiale, D Lacour, F Montaigne, M. Hehn

\section{- To cite this version:}

J Torrejon, Grégory Malinowski, M Pelloux, R Weil, A Thiaville, et al.. Unidirectional Thermal Effects in Current-Induced Domain Wall Motion. Physical Review Letters, 2012, 109, pp.106601. 10.1103/PhysRevLett.109.106601 . hal-02949044

\section{HAL Id: hal-02949044 \\ https://hal.science/hal-02949044}

Submitted on 25 Sep 2020

HAL is a multi-disciplinary open access archive for the deposit and dissemination of scientific research documents, whether they are published or not. The documents may come from teaching and research institutions in France or abroad, or from public or private research centers.
L'archive ouverte pluridisciplinaire HAL, est destinée au dépôt et à la diffusion de documents scientifiques de niveau recherche, publiés ou non, émanant des établissements d'enseignement et de recherche français ou étrangers, des laboratoires publics ou privés. 


\title{
Unidirectional Thermal Effects in Current-Induced Domain Wall Motion
}

\author{
J. Torrejon, ${ }^{*}$ G. Malinowski, M. Pelloux, R. Weil, and A. Thiaville \\ Laboratoire de Physique des Solides, Université Paris-Sud, CNRS UMR 8502, 91405 Orsay, France
}

\section{J. Curiale}

Laboratoire de Physique des Solides, Université Paris-Sud, CNRS UMR 8502, 91405 Orsay, France and Consejo Nacional de Investigaciones Científicas y Técnicas, Centro Atómico Bariloche-Comisión Nacional de Energía Atómica, Avenida Bustillo 9500, 8400 San Carlos de Bariloche, Río Negro, Argentina ${ }^{\dagger}$

\author{
D. Lacour, F. Montaigne, and M. Hehn \\ Institut Jean Lamour, Université Nancy I, 54506 Vandoeuvre-lès-Nancy, France
}

(Received 11 May 2012; published 4 September 2012)

\begin{abstract}
We report experimental evidence of thermal effects on the displacement of vortex walls in $\mathrm{NiFe}$ nanostrips. With the use of nanosecond current pulses, a unidirectional motion of the magnetic domain walls towards the hotter part of the nanostrips is observed, in addition to current-induced domain wall motion. By tuning the heat dissipation in the samples and modeling the heat diffusion, we conclude that this unidirectional motion can only be explained by the presence of a temperature profile along the nanostrip. A quantitative analysis of the experiments shows that, on top of the classical thermodynamic pressure on the domain wall, another force, probably the magnonic spin Seebeck effect, is displacing the domain walls.
\end{abstract}

DOI: 10.1103/PhysRevLett.109.106601

PACS numbers: 72.20.Pa, 75.30.Ds, 75.76.+j, 75.78.Fg

The consideration of thermal effects in spintronics has opened a new avenue of research [1]. The induction of a voltage by a temperature difference- the Seebeck effecthas been known since the early days of solid-state physics. In the two-current model of Mott for magnetic metals, a spin dependence of the Seebeck coefficient is expected, leading to a thermoelectric spin potential, the spindependent Seebeck effect [2]. However, similar observations in insulating ferromagnets have forced considering other microscopic origins, such as magnonic spin Seebeck effects [3-5] due to the spin current linked to magnons flow, in strong coupling with nonequilibrium phonons [6]. This multitude of effects calls for experiments that weigh their relative magnitudes. Such an identification is especially important for nanosciences, as temperature gradients that are impossible in bulk samples are easily created in nanostructures.

The spin Seebeck effects can be evidenced either in uniformly magnetized systems, through induced voltages [2] or, as proposed in several papers [5,7-10], by a displacement of a magnetic boundary between domains with opposite magnetizations, i.e., a magnetic domain wall (DW). Despite early work on infinite films by Berger and co-workers [11,12], unambiguous experimental tests of the proposed effects on nanostructures are still very scarce [13]. Note that, in bulk samples, additional effects are also present, such as the generation of eddy currents around the DW due to the Nernst effect [11]. In this Letter, we introduce a new configuration to test the influence of thermal effects on DW in nanostructures (Fig. 1). By injecting a nanosecond current pulse into a magnetic nanostrip, the temperature is locally and instantaneously raised to a high and nearly uniform value, mainly controlled by the nature of the multilayer from which the nanostrip was patterned. In addition, the DW present in the nanostrip is set into motion under the action of the spin polarization of the current, by the spin transfer torque (STT, see Ref. [14] for a recent review). In the cooling phase, a large transient temperature gradient appears along the length of the nanostrip. We observe that this gives rise to a unidirectional force pushing the DW towards the hotter part-the center - of the nanostrip. This motion adds to or reduces

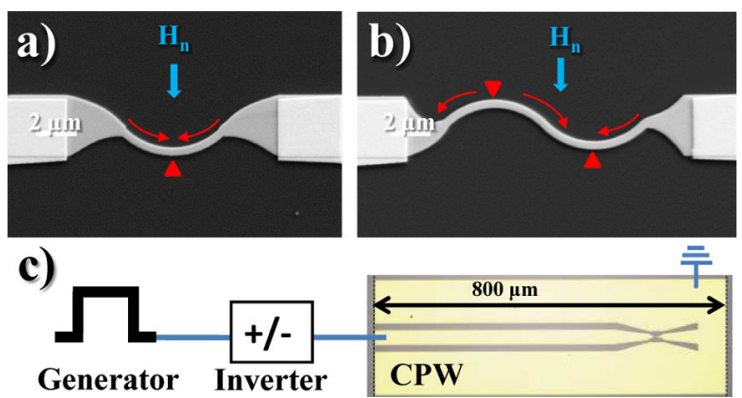

FIG. 1 (color online). Schematic of the experiment and sample structures. (a) SEM image of a single-curve sample, in which one DW is nucleated at the center by a transverse field $H_{n}$. (b) In the double-curve case, this procedure creates two DWs at $1 / 4$ and $3 / 4$ of the length. (c) Optical image of the coplanar waveguide by which the sample is connected, with a schematic of the electronics. 
the DW displacement due to STT, whose direction is fixed by current polarity. The net DW displacement long after the pulse is imaged by high-resolution magnetic force microscopy (MFM), the experiment being performed in situ (see Ref. [15] for the description of the setup).

Effects of sample heating, due to the large current densities required for observing DW displacement by STT, have been reported by many authors. The nucleation of domains [16-18] has been attributed to an increase of the sample temperature above the Curie temperature, whereas DW structure transformation and/or random displacement have been related to thermally activated Brownian motion of the wall position and magnetic moment $[19,20]$. The effect reported here is qualitatively different, as a unidirectional DW displacement towards the hotter part of the nanostrip is observed, irrespective of the current direction. Remarkably, this directionality is opposite to that observed on bulk samples [12].

Magnetically soft $\mathrm{Ta}(3 \mathrm{~nm}) / \mathrm{Ni}_{81} \mathrm{Fe}_{19}(17 \mathrm{~nm}) / \mathrm{Pt}(3 \mathrm{~nm})$ thin films were deposited onto Si substrates with or without a $100 \mathrm{~nm} \mathrm{SiO}_{2}$ layer, using UHV sputtering. By nanopatterning, nanostrips with a width varying between 200 and $500 \mathrm{~nm}$ and a length of 6 or $12 \mu \mathrm{m}$ were obtained. They were designed with a single or double curvature to easily nucleate one or two DWs by applying a strong inplane field, in a direction transverse to the nanostrip [Figs. 1(a) and 1(b)]. The $\mathrm{Ti}(5 \mathrm{~nm}) / \mathrm{Au}(70 \mathrm{~nm})$ contact electrodes belong to a coplanar waveguide, the nanostrip connecting ground and signal [Fig. 1(c)].

Typical experimental results are illustrated by the MFM images of Fig. 2, obtained on double curvature samples with two DWs. For the sample grown on a Si substrate with a thick insulating silicon oxide layer [Fig. 2(a)], the displacement of the DW towards the center of the nanostrip (red dashed line) is distinctly easier compared to the motion towards the extremities. The experiments, repeated for the opposite DW magnetostatic charges (by changing the sense of the in-plane nucleation field), yield identical results, proving that no parasitic field compressing the central domain is present. An effect of a pinning site can be excluded since the initial DW position is different for each experiment. Indeed, the nucleated DW is an (asymmetric) transverse wall [21], metastable in the present samples. On the first pulse, this DW transforms to the stable vortex wall (VW) structure, with an added displacement due to the automotion effect that shows some scatter [15]. The experiment was also reproduced on several samples, with identical or different nanostrip widths. In contrast [Fig. 2(b)], for an identical structure grown on $\mathrm{Si}$ substrate with only a native silicon oxide, no asymmetry is observed, in agreement with experiments on similar structures [15]: large and reproducible displacements of about $800 \mathrm{~nm}$ are observed (for $3 \mathrm{~ns}$ pulses).

The difference between the two directions of motion, towards or away from the center, in the case of the

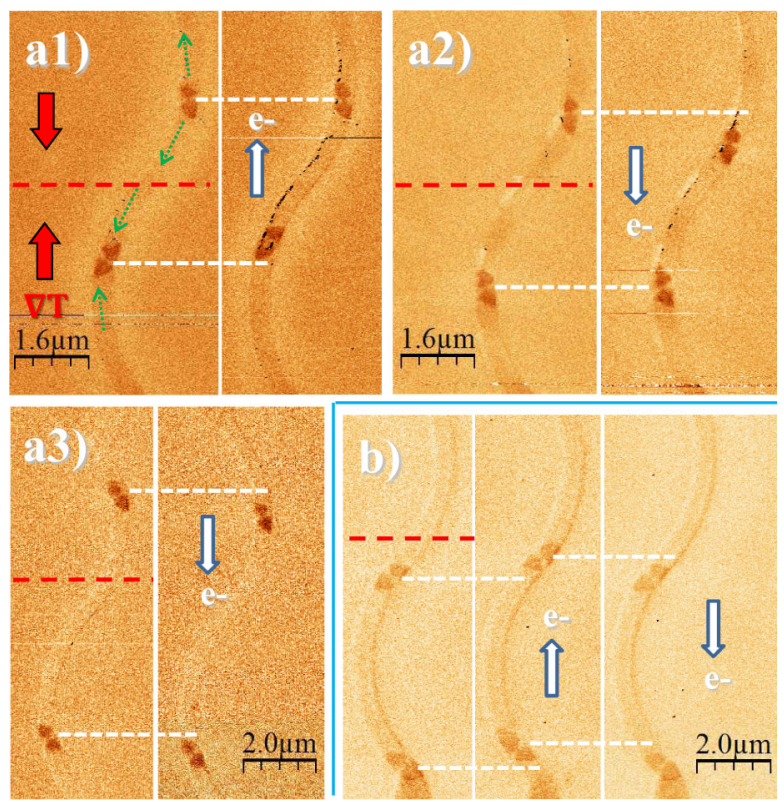

FIG. 2 (color online). VW motion observed by MFM in a nanostrip with double curvature, $450 \mathrm{~nm}$ wide and $12 \mu \mathrm{m}$ long. The upper (lower) VW corresponds to the tail to tail (head to head) configuration (green arrows with dotted-line tails schematize the magnetization orientation). Current pulses of $2 \mathrm{~ns}$ duration and $3 \mathrm{TA} / \mathrm{m}^{2}$ amplitude (open arrows display the electrons' motion) are applied between consecutive MFM images. The temperature gradient, indicated by red full arrows, is towards the center (red dashed line). Typical results for a nanostrip grown on a $100 \mathrm{~nm}$ oxide layer are shown as follows: (a1) electrons injected from bottom, motion of lower VW by $400 \mathrm{~nm}$; (a2) electrons injected from the top, motion of upper VW by $800 \mathrm{~nm}$; (a3) rarely observed motion of both VW, but still with a larger displacement towards the center. For an identical sample with native silicon oxide (b), symmetrical motion in both directions is observed ( $3 \mathrm{~ns}$ pulses).

thick oxide samples, was observed both on the motion probability and on the average displacement. In order to quantify the effect, the experiment was repeated many times to construct displacement histograms. Figure 3 shows typical results, obtained for a nanostrip with width $w=450 \mathrm{~nm}$, for two current densities at constant $2 \mathrm{~ns}$ pulse duration. The total probability of motion is much higher when STT drives the DW towards the center part of the sample $(62.5 \%)$ compared to the opposite direction $(20 \%)$. Moreover, it is clear that the displacement is also much larger when the DW motion takes place toward the center of the nanostrip. In comparison, histograms for the sample with only a native oxide are symmetrical [22].

Changing the substrate for the growth of the metallic multilayer has several consequences. The DW pinning is more important in the thick oxide case, with wider histograms and larger no-motion probability. However, this should not favor DW motion in any direction of the nanostrip. Another obvious consequence of a thick oxide is the reduced cooling by the substrate of the nanostrip 


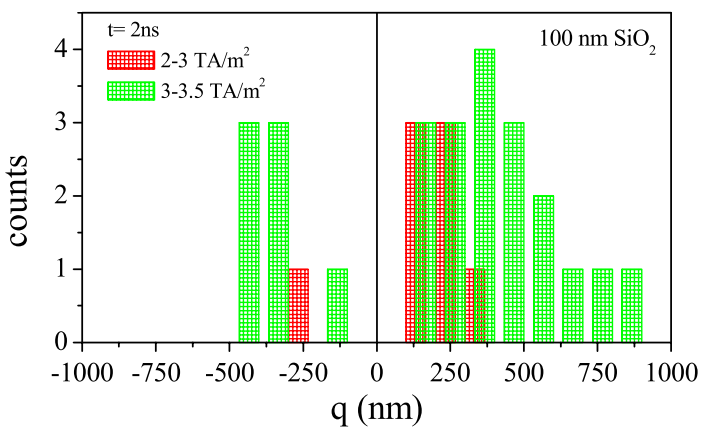

FIG. 3 (color online). Histogram of measured nonzero VW displacements, $q$, for a nanostrip of width $w=450 \mathrm{~nm}$ grown on thick silicon oxide. Positive (negative) values correspond to the propagation towards the center of nanostrip (resp. ends). As there are 20 trials for each current pulse category and polarity, the number of no-motion results, not shown at $q=0$, can be obtained by difference.

submitted to Joule heating [23]. For example, we compute with a $2 \mathrm{D}$ calculation that after $2 \mathrm{~ns}$ with a current density of $2 \mathrm{TA} / \mathrm{m}^{2}$, with $20 \mu \Omega \cdot \mathrm{cm} \mathrm{NiFe}$ resistivity, the temperature in a $400 \times 20 \mathrm{~nm}^{2}$ nanostrip of infinite length has increased from $T_{0}=300 \mathrm{~K}$ to $T=353 \mathrm{~K}$ with a native $2 \mathrm{~nm}$ oxide, and up to $527 \mathrm{~K}$ for a $100 \mathrm{~nm}$ oxide [22]. Thus, the Curie temperature is easily reached with a thick oxide, and indeed the nucleation of domains or a uniform remagnetization were observed for too intense or too long pulses. However, a uniform temperature increase over the entire length of the nanostrip does not by itself lead to an effective force on the DW. On the contrary, the weak variation of DW magnetostatic energy due to the finite nanostrip length that pushes the DW out of the center decreases at high temperature. Similarly, considering thermal activation, one only expects that an increased temperature favors DW motion down its magnetostatic potential well, thus, towards the ends. Therefore, the only plausible explanation to account for our observations relies on the presence of a temperature gradient along the nanostrip.

As stressed recently, the largest gradient in such geometry is perpendicular to the film plane [24]. Numerical calculations for both a native oxide and a thick oxide layer of $100 \mathrm{~nm}$ give temperature gradients of $\partial T / \partial z=340$ and $170 \mathrm{~K} / \mu \mathrm{m}$ for a $2 \mathrm{~ns}, 2 \mathrm{TA} / \mathrm{m}^{2}$ current pulse. Therefore, a larger gradient is present in the absence of oxide, reflecting only a better heat dissipation into the substrate, in qualitative contradiction with observations. Moreover, the thermoelectric effect that has been mentioned in such case $[12,24]$ is the anomalous Nernst effect. A coefficient $\chi=0.13$ between the anomalous and Seebeck effects has been recently measured in $\mathrm{NiFe}$ [25]. With the nominal $\mathrm{NiFe}$ resistivity, this corresponds to an induced in-plane current density of magnitude $2.7 \mathrm{GA} / \mathrm{m}^{2}$, locally perpendicular to the magnetization. This magnitude is nearly 1000 times smaller than the injected current density, and moreover the STT from this Nernst current would have a zero average for a VW. As a result, the perpendicular temperature gradient cannot explain the observed effect.

We therefore consider a longitudinal temperature gradient. During the pulse, as it is extremely short, heat cannot diffuse out of the nanostrip. As a result, the temperature over the full length of the nanostrip is very homogeneous even at the end of the pulse (from the quoted value of the $\mathrm{NiFe}$ thermal conductivity [25] a heat diffusion constant $D \approx 8 \mathrm{~mm}^{2} / \mathrm{s}$ is estimated, leading to $\sqrt{D t} \approx 100 \mathrm{~nm}$ at $t=1 \mathrm{~ns})$. On the other hand, the cooling lasts much longer, so that a nonuniform temperature profile can develop as a competition between heat absorption by the substrate and by the thick Au contacts at the nanostrip ends. The increased heat dissipation at the nanostrip ends leads to a larger temperature at the nanostrip center. In fact, the sample with thick oxide favors such a gradient, with a higher initial temperature and a much lower substrate heat conduction The cooling process lasts longer, from a few ns (native oxide) to $50 \mathrm{~ns}$ (100 nm thick oxide). The temperature profiles were evaluated with 3D timedependent finite element modeling [22]. The results show that a "temperature wave" sweeps the two halves of the nanostrip during cooling. These calculations, performed with tabulated parameters, give an order of magnitude of the longitudinal temperature gradient expected in such conditions. Large values above $100 \mathrm{~K} / \mu \mathrm{m}$ are calculated initially and close to the nanostrip extremities whereas almost negligible values, less than $1 \mathrm{~K} / \mu \mathrm{m}$, are obtained at long times in the central part.

What are the forces exerted by a temperature gradient on a DW? A spin transfer force due to the spin-dependent Seebeck effect has been considered recently [7,9,26,27]. Similarly to conventional STT induced by current, it involves a nonadiabatic $\beta$-like parameter, whose value is not well-known. However, with the negative sign of the Seebeck coefficient of $\mathrm{NiFe}$ and a positive sign for $\beta$, this torque will push the DW towards the cold part [27], opposite to our observations. In addition, the latter effect is relatively weak as one computes for a $100 \mathrm{~K} / \mu \mathrm{m}$ gradient an equivalent charge current density of $10 \mathrm{GA} / \mathrm{m}^{2}$ only (resistivity $20 \mu \Omega \cdot \mathrm{cm}$, Seebeck coefficient $-20 \mu \mathrm{V} / \mathrm{K}$ ), more than 100 times smaller than the injected current density.

A thermodynamic force, the gradient of DW energy caused by the temperature gradient, has been known for a long time [28]. As all magnetic energies disappear at the Curie temperature, it is expected to push the DW towards the hotter part [5], as observed here. In a mean-field model for the temperature dependence of magnetization, the DW energy in a soft material decreases linearly with temperature down to the Curie temperature. Thus, an effective field proportional to the temperature gradient exists $\vec{H}_{T}=\vec{\nabla} T \sigma_{0} /\left[2 \mu_{0} M_{s}\left(T_{c}-T_{0}\right)\right]$, with $M_{s}$ the spontaneous magnetization, and $\sigma_{0}$ the DW surface energy at the experiment temperature $T_{0} \quad\left(T_{c}\right.$ being the Curie 
temperature). Assuming that the DW is not pinned and does not change structure, the DW displacement during the cooling phase is then simply [15] $q=\left(\gamma_{0} \Delta_{0} / \alpha\right) \times$ $\int H_{T}(t) d t$, where the value of the effective thermodynamic field has to be taken along the DW trajectory, $\gamma_{0}$ is the gyromagnetic ratio, $\Delta_{0}$ the DW width, and $\alpha$ the Gilbert damping constant of magnetization dynamics. From the computed 50 aJ DW energy and damping constant $\alpha=0.01$, we expect a thermodynamic DW displacement of $q \approx 2000 \mathrm{~nm}$ close to the ends of nanostrip, for a $2 \mathrm{TA} / \mathrm{m}^{2}, 2 \mathrm{~ns}$ pulse. In order to obtain a more precise evaluation of the DW displacement, taking into account STT, thermodynamic field, and automotion effects, a numerical solution of the 1D model has been implemented [22]. This model describes the VW dynamics by two conjugate coordinates, corresponding to the longitudinal position of the core and the (generalized) DW magnetization angle, proportional here to the transversal position of the vortex core. It has been shown to properly depict the VW dynamics under current excitation [29]. Indeed, as the relaxation time of the VW structure is long $(\approx 14 \mathrm{~ns}$ here), it is comparable to the cooling time of the nanostrip so that the VW displacements induced by STT and thermodynamic effective field cannot be simply added. The results of such a calculation are superposed to the measurements in Fig. 4(a). For this second series of experiments, the DW was deliberately placed at all positions along the nanostrip by means of an additional in-plane field. Note that, close to the nanostrip ends, VW structure transformations or even DW expulsion were sometimes observed, leading to a lower density of measured displacements. The calculations qualitatively reproduce the data and also show VW transformation in the vicinity of the nanostrip ends, as seen by the large automotion linked to DW structure transformation [15]. Thus, although full micromagnetic simulations are required for fully estimating the effects of such a temperature wave, we see that the combination of STT and thermodynamic DW motion explains the general trend of the data.

However, a significant difference appears. Contrary to the case in thermodynamic calculations, the DWs were not observed to move freely under STT in the central part of the sample, especially in the case where motion due to STT and to the thermodynamic force are in competition. The reason for this prediction is clear from the normalized temperature profiles shown in Fig. 4(b): even at $6 \mu \mathrm{m}$ length, there is virtually no temperature gradient in the vicinity $( \pm 1 \mu \mathrm{m})$ of the nanostrip center, hence no thermodynamic effective field acting on the DW. Recent experiments have underlined the role of magnons in thermoelectric effects $[3,4,30]$. We note that the predictions about the direction of DW motion under magnon flow are of conflicting signs, either in the direction of the magnons flow [31] or towards the hot part of the sample $[5,32,33]$, with no experimental proof up to now. Despite

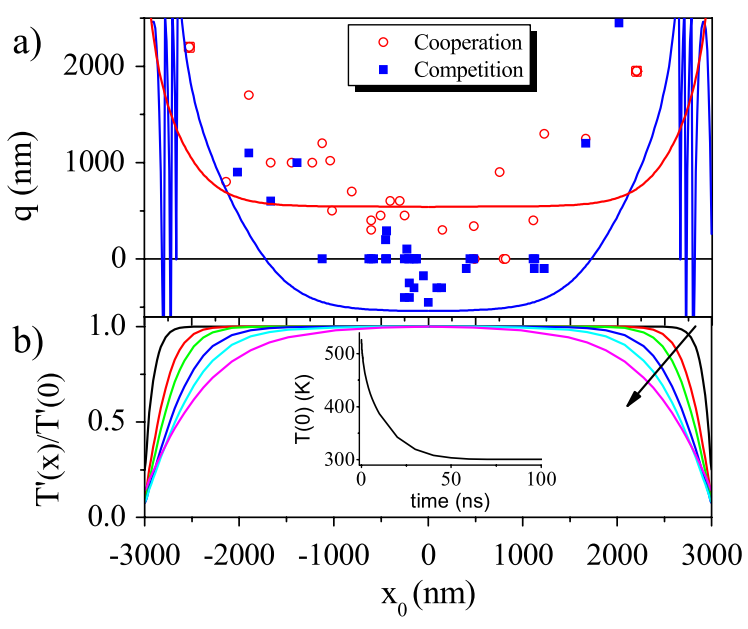

FIG. 4 (color online). (a) Measured VW displacement as a function of initial position $\left(x_{0}\right)$, after injecting a current pulse, $2 \mathrm{~ns}$ long and with $2 \mathrm{TA} / \mathrm{m}^{2}$ amplitude, for single curvature nanostrips $400 \mathrm{~nm}$ wide and $6 \mu \mathrm{m}$ long. Positive displacement refers to motion towards the nanostrip center, and open (full) symbols correspond to STT driving the VW to the center (resp. end). The curves show the values calculated using a 1D model with two collective coordinates, including STT and the thermodynamic effective field, for a damping constant $\alpha=0.01$. (b) Normalized temperature increase $T^{\prime}=$ $T-T_{0}$ profiles computed for times $0,5,10,20,30$, and 48 ns after the current pulse end (arrow shows the effect of time increase). The decrease of central temperature $T(0, t)$ with time $t$ is shown in inset. See Supplemental Material [22] for parameters.

this, an interesting property of magnons is that they can transport information over micrometer-long distances, thus even in the absence of a local temperature gradient $[3,5]$. Therefore, it appears that the thermal effect observed on the DW motion close to the nanostrip center is likely to be explained by a magnonic spin Seebeck effect.

In conclusion, we have shown that, in nanoscale samples where the heat flow is restricted so that planar temperature gradients result from nanosecond current pulses, a large thermal displacement of magnetic DWs occurs. This displacement, towards the hot part of the sample, is distinct from Brownian motion due to global sample heating. It increases or decreases the displacement of the wall due to spin-transfer torque. Considering the magnitude and direction of this displacement, we have shown that neither the anomalous Nernst effect nor the electronic (spin) Seebeck effect can explain it. On the other hand, a thermodynamic model explains a large part of the effect, leaving unexplained, however, the DW displacement observed in regions with no temperature gradient but surrounded (at $\approx 1 \mu \mathrm{m}$ ) by lower temperature regions. We ascribe it to magnonic spin Seebeck effect and conclude that the action of magnons on domain walls DWs in a very nonuniform temperature landscape deserves further investigation so as to become quantitative. 
J.T. acknowledges the support by the European Community's Seventh Framework Programme, through an individual IEF Marie Curie fellowship (CIDWMnanostrips, No. 251887). The research at LPS was partially supported by the French Agence Nationale de la Recherche, Project No. HYFONT ANR-09-NANO-002.

*torrejon@1ps.u-psud.fr

${ }^{\dagger}$ Also at Instituto Balseiro, Comisión Nacional de Energía Atómica and Universidad Nacional de Cuyo, Avenida Bustillo 9500, 8400 S. C. de Bariloche, RN, Argentina.

[1] G. E. W. Bauer, E. Saitoh, and B. J. van Wees, Nature Mater. 11, 391 (2012).

[2] K. Uchida, S. Takahashi, K. Harii, J. Ieda, W. Koshibae, K. Ando, S. Maekawa, and E. Saitoh, Nature (London) 455 , 778 (2008).

[3] J. Xiao, G. E. W. Bauer, K. C. Uchida, E. Saitoh, and S. Maekawa, Phys. Rev. B 81, 214418 (2010).

[4] J. C. Slonczewski, Phys. Rev. B 82, 054403 (2010).

[5] D. Hinzke and U. Nowak, Phys. Rev. Lett. 107, 027205 (2011).

[6] H. Adachi, K. Uchida, E. Saitoh, J. Ohe, S. Takanashi, and S. Maekawa, Appl. Phys. Lett. 97, 252506 (2010).

[7] A. A. Kovalev and Y. Tserkovnyak, Phys. Rev. B 80, 100408(R) (2009).

[8] K. M. D. Hals, A. Brataas, and G. E. W. Bauer, Solid State Commun. 150, 461 (2010).

[9] Z. Yuan, S. Wang, and K. Xia, Solid State Commun. 150, 548 (2010).

[10] G. E. W. Bauer, A. H. MacDonald, and S. Maekawa, Solid State Commun. 150, 459 (2010).

[11] S. U. Jen and L. Berger, J. Appl. Phys. 59, 1278 (1986).

[12] S. U. Jen and L. Berger, J. Appl. Phys. 59, 1285 (1986).

[13] P. Möhrke, J. Rhensius, J.-U. Thiele, L. Heydermann, and M. Kläui, Solid State Commun. 150, 489 (2010).

[14] O. Boulle, G. Malinowski, and M. Kläui, Mater. Sci. Eng. R 72, 159 (2011).

[15] J.-Y. Chauleau, R. Weil, A. Thiaville, and J. Miltat, Phys. Rev. B 82, 214414 (2010).

[16] A. Yamaguchi, S. Nasu, H. Tanigawa, T. Ono, K. Miyake, K. Mibu, and T. Shinjo, Appl. Phys. Lett. 86, 012511 (2005).
[17] Y. Togawa, T. Kimura, K. Harada, T. Akashi, T. Matsuda, A. Tonomura, and Y. Otani, Jpn. J. Appl. Phys. 45, L683 (2006).

[18] M. Cormier, A. Mougin, J. Ferré, A. Thiaville, N. Charpentier, F. Piéchon, R. Weil, V. Baltz, and B. Rodmacq, Phys. Rev. B 81, 024407 (2010).

[19] M. Laufenberg, W. Bührer, D. Bedau, P.-E. Melchy, M. Kläui, L. Vila, G. Faini, C. A.F. Vaz, J. A.C. Bland, and U. Rüdiger, Phys. Rev. Lett. 97, 046602 (2006).

[20] F. Junginger, M. Kläui, D. Backes, U. Rüdiger, T. Kasama, R. E. Dunin-Borkowski, L. J. Heyderman, C. A. F. Vaz, and J. A. C. Bland, Appl. Phys. Lett. 90, 132506 (2007).

[21] M. Kläui, C. A. F. Vaz, J. A. C. Bland, L. J. Heyderman, F. Nolting, A. Pavlovska, E. Bauer, S. Cherifi, S. Heun, and A. Locatelli, Appl. Phys. Lett. 85, 5637 (2004).

[22] See Supplemental Material at http://link.aps.org/ supplemental/10.1103/PhysRevLett.109.106601 for additional wall displacement histograms, description of heat diffusion calculations procedure and results, and presentation of the micromagnetic simulations and 1D model calculations that have been performed.

[23] C.-Y. You and S.-S. Ha, Appl. Phys. Lett. 91, 022507 (2007).

[24] S. Y. Huang, W. G. Wang, S. F. Lee, J. Kwo, and C. L. Chien, Phys. Rev. Lett. 107, 216604 (2011).

[25] A. Slachter, F. L. Bakker, and B. J. van Wees, Phys. Rev. B 84, 020412(R) (2011).

[26] M. Hatami, G. E. W. Bauer, Q. Zhang, and P. J. Kelly, Phys. Rev. Lett. 99, 066603 (2007).

[27] G.E.W. Bauer, S. Bretzel, A. Brataas, and Y. Tserkovnyak, Phys. Rev. B 81, 024427 (2010).

[28] A. Thiele, A. Bobeck, E. Della Torre, and U. Gianola, Bell Syst. Tech. J. 50, 711 (1971).

[29] L. Thomas, M. Hayashi, X. Jiang, R. Moriya, C. Rettner, and S. S. P. Parkin, Nature (London) 443, 197 (2006).

[30] Y. Kajiwara, K. Harii, S. Takahashi, J. Ohe, K. Uchida, M. Mizuguchi, H. Umezawa, H. Kawai, K. Ando, K. Takanashi, S. Maekawa, and E. Saitoh, Nature (London) 464, 262 (2010).

[31] D.-S. Han, S.-K. Kim, J.-Y. Lee, S. J. Hermsdoerfer, H. Schultheiss, B. Leven, and B. Hillebrands, Appl. Phys. Lett. 94, 112502 (2009).

[32] A. V. Mikhailov and A. I. Yaremchuk, JETP Lett. 39, 354 (1984).

[33] P. Yan, X. S. Wang, and X. R. Wang, Phys. Rev. Lett. 107, 177207 (2011). 